\title{
GROUPS OF BREADTH FOUR HAVE CLASS FIVE
}

\author{
by I. D. MACDONALD
}

(Received 23 February, 1977)

A conjecture of reputable vintage states that $c(G) \leq b(G)+1$ for a finite $p$-group $G$ of class $c(G)$ and breadth $b(G)$. This result has been proved in a medley of special cases and in particular whenever $b(G) \leq 3$. We now prove it for $b(G)=4$.

1. Introduction. Let $G$ be a finite $p$-group and let $C(x)$ denote the centraliser of the element $x$ in $G$. The breadth $b(x)$ is defined by

$$
p^{b(x)}=|G: C(x)|,
$$

and the breadth $b=b(G)$ of $G$ is defined by

$$
b=b(G)=\max \{b(x): x \in G\} .
$$

Discussion of the conjecture that the class $c(G)$ of the finite $p$-group $G$ is bounded by $b(G)+1$ may be found in the references, especially [6] and [7]. The result for $b(G)=1$ was proved by Burnside [1,pp. 125-6]. Knoche [5] proved that $c(G) \leq b(G)+1$ for $2 \leq b(G) \leq 3$. Other special cases have been studied. The general case may yet prove to be false. The results of [7] suggest that the cases with $b(G) \leq 6$ may well be decisive because counter-examples with $b(G)=6$ are there presented to settle certain closely-related conjectures, which appear as Problems 3.5 and 3.6 in [6].

In this note we show that well-tried methods suffice to settle the case $b(G)=4$. More precisely we prove:

THEOREM. If $G$ is a finite p-group with $b(G)=4$ then $c(G) \leq 5$.

2. Definitions and preliminaries. To prove the theorem we need the apparatus of commutator manipulation. In this section we survey the necessary equipment and, to give a taste of the argument, apply the methods to the case $b(G)=3$.

Let $x_{1}, x_{2}, \ldots, x_{n}, \ldots$ be elements of the group $G$. Then

$$
\left[x_{1}, x_{2}\right]=x_{1}^{-1} x_{2}^{-1} x_{1} x_{2}
$$

and if $n \geq 2$ then

$$
\left[x_{1}, x_{2}, \ldots, x_{n+1}\right]=\left[\left[x_{1}, x_{2}, \ldots, x_{n}\right], x_{n+1}\right]
$$

We use the "semicolon notation", according to which

$$
\begin{gathered}
{\left[x_{1}, \ldots, x_{p} ; x_{p+1}, \ldots, x_{p+q}\right]=\left[c_{p}, c_{q}\right]} \\
{\left[x_{1}, \ldots, x_{p} ; x_{p+1}, \ldots, x_{p+q} ; x_{p+q+1}, \ldots, x_{p+q+r}\right]=\left[c_{p}, c_{q}, c_{r}\right]}
\end{gathered}
$$

Glasgow Math. J. 19 (1978) 141-148 
and so on, where

$$
c_{p}=\left[x_{1}, \ldots, x_{p}\right], c_{q}=\left[x_{p+1}, \ldots, x_{p+q}\right], c_{r}=\left[x_{p+q+1}, \ldots, x_{p+q+r}\right] .
$$
and

The terms $\gamma_{i}(G)$ of the lower central series of $G$ are defined by putting $\gamma_{1}(G)=G$

$$
\gamma_{i}(G)=\left\langle\left[x_{1}, \ldots, x_{i}\right]: x_{1} \in G, \ldots, x_{i} \in G\right\rangle
$$

for $i>1$. The group $G$ is said to have class $n=c(G)$ if $\gamma_{n+1}(G)=1$ but $\gamma_{n}(G) \neq 1$. The two-step centralisers $C_{i}$ corresponding to the lower central series are defined for $1 \leq i<c$ by

$$
C_{i}=\left\langle x \in G: \text { if } y \in \gamma_{i}(G) \text { then }[x, y] \in \gamma_{i+2}(G)\right\rangle .
$$

Notice that $C_{i} \neq G$, and that $C_{i}$ is normal in $G$, for $1 \leq i<c$.

Next we summarise some useful results. The multilinearity property of commutators will be referred to (if at all) as (ML), at its frequent appearances; we are thinking of statements like

$$
\left[y_{1} z_{1}, x_{2}, \ldots, x_{n}\right] \equiv\left[y_{1}, x_{2}, \ldots, x_{n}\right]\left[z_{1}, x_{2}, \ldots, x_{n}\right] \bmod \gamma_{n+1} .
$$

(In fact what $\equiv$ really denotes in statements like $u \equiv v \bmod \gamma_{n+1}$ or $u \equiv v \bmod \gamma_{n+1}(G)$ is that the cosets $u \gamma_{n+1}(G)$ and $v \gamma_{n+1}(G)$ in $G / \gamma_{n+1}(G)$ are equal.)

We denote by (JW) the result of a standard identity, namely

$$
\left[c_{p}, c_{q}, c_{r}\right]\left[c_{q}, c_{r}, c_{p}\right]\left[c_{r}, c_{p}, c_{q}\right] \equiv 1 \bmod \gamma_{p+q+r+1}
$$

or

$$
\left[c_{p},\left[c_{q}, c_{r}\right]\right] \equiv\left[c_{p}, c_{q}, c_{r}\right]\left[c_{p}, c_{r}, c_{q}\right]^{-1} \bmod \gamma_{p+q+r+1}
$$

where $c_{p}, c_{q}, c_{r}$ are defined as above.

Two consequences of (JW) are helpful. The first, (LN), states that a commutator of the form $\left[x_{1}, \ldots, x_{n}\right]$ is the product of commutators like $\left[x_{n}, y_{1}, \ldots, y_{n-1}\right]$ and its inverse, $\bmod \gamma_{n+1}$, where $\left\{y_{1}, \ldots, y_{n-1}\right\}=\left\{x_{1}, \ldots, x_{n-1}\right\}$. The second is denoted by $(A B)$ :

$$
\left[x_{1}, x_{2}, x_{1}, x_{2}\right] \equiv\left[x_{1}, x_{2}, x_{2}, x_{1}\right] \bmod \gamma_{5} \text {. }
$$

Now consider a finite $p$-group $G$ with breadth $b$ and class $c>b+1$. We can make a reduction by replacing $G$ with $G / \gamma_{b+3}(G)$, for an obvious inductive assumption allows us to suppose that the breadth of $G / \gamma_{b+3}(G)$ is not less than $b$. In other words, in proving the theorem we may take $c(G)=b(G)+2$.

Lemma 3.1 of [6] shows that $C_{1} \cup \ldots \cup C_{c-1} \neq G$ implies that $c \leq b+1$. But $C_{1} \leq C_{i}$ for $1<i<c$, as (LN) clearly implies. We shall prove that if $3 \leq b \leq 4$ then $c \leq b+1$ by first establishing: if $3 \leq b \leq 4$ and $c=b+2$ then $C_{2} \cup \ldots \cup C_{b+1} \neq G$.

Let us consider the case of a finite p-group $G$ with $b(G)=3$ and $c(G)=5$, and let us suppose that $G=C_{2} \cup C_{3} \cup C_{4}$. It is a known fact that if $D=C_{2} \cap C_{3} \cap C_{4}$ then $G / D$ is non-cyclic of order 4 , and though this is due to Scorza (1926), according to [3], a more convenient reference is [4]. 
Therefore $p=2, G=\langle a, b, D\rangle, C_{2}=\langle a, D\rangle, C_{3}=\langle b, D\rangle, C_{4}=\langle a b, D\rangle$. Let $x_{1}, \ldots, x_{5}$ be elements of $G$ for which $w=\left[x_{1}, \ldots, x_{5}\right] \neq 1$. Clearly $D$ does not contain $x_{3}$ or $x_{4}$ or $x_{5}$, and without losing generality we may take $x_{3}=b, x_{4}=a$. If we work $\bmod \gamma_{5}(G)$ then

$$
\left[x_{1}, x_{2}, b, a\right] \equiv\left[x_{1}, x_{2} ; b, a\right]\left[x_{1}, x_{2}, a, b\right] \quad(\mathrm{JW}) \text {. }
$$

But $\left[x_{1}, x_{2}, a, b\right] \equiv 1$ since for instance $a \in C_{2}$. Further

$$
\left[x_{1}, x_{2} ; b, a\right] \equiv\left[a, b ; x_{1}, x_{2}\right]
$$

and another application of (JW), whose details we suppress, shows that if $x_{1}$ or $x_{2}$ lies in $D$, and so in both $C_{2}$ and $C_{3}$, then $\left[a, b ; x_{1}, x_{2}\right] \equiv 1$. So we may assume that $x_{1}=a, x_{2}=b$, in which case by $(\mathrm{AB})$,

$$
\left[x_{1}, x_{2}, x_{3}, x_{4}\right]=[a, b, b, a] \equiv[a, b, a, b] .
$$

Since $a \in C_{2}$, it follows that $w=1$. This is a contradiction, and shows that $G \neq C_{2} \cup C_{3} \cup$ $C_{4}$. The result that if $b=3$ then $c \leq 4$ follows as explained above.

3. Proof of the theorem: the redundant case. In order to prove the theorem we suppose that $G$ is a finite $p$-group with $b(G)=4, c(G)=6$, and $G=C_{2} \cup C_{3} \cup C_{4} \cup C_{5}$. The calculations in this case will be presented in a more succinct form than above.

A complication immediately arises, for $G$ may be the union of just three of the proper subgroups $C_{i}(2 \leq i \leq 5)$, and disposing of this case is not trivial. Consideration of subcases is necessary. We always suppose that $w \neq 1$ where

$$
w=\left[x_{1}, x_{2}, x_{3}, x_{4}, x_{5}, x_{6}\right] \text {. }
$$

(i) Suppose that $G=C_{3} \cup C_{4} \cup C_{5}$. Put $D=C_{3} \cap C_{4} \cap C_{5}, C_{3}=\langle a, D\rangle, C_{4}=\langle b, D\rangle$, $C_{5}=\langle a b, D\rangle$. We take $x_{4}=b, x_{5}=a, x_{6}=a$.

Our first aim is to show that no $x_{i}$ lies in $D$. By (LN), if some $x_{i} \in D$ then we can take $i=1$. We have

and

$$
\left[x_{1}, x_{2}, x_{3}, b, a\right] \equiv\left[x_{1}, x_{2}, x_{3} ; b, a\right] \quad\left(\mathrm{JW} ; a \in C_{3}\right)
$$

$$
\begin{aligned}
w & =\left[x_{1}, x_{2}, x_{3} ; b, a ; a\right] \\
& =\left[a, b, a ; x_{1}, x_{2}, x_{3}\right]\left[x_{1}, x_{2}, x_{3}, a ; b, a\right] \quad(\mathrm{JW}) .
\end{aligned}
$$

The former of these commutators is trivial because $x_{1} \in C_{3} \cap C_{4} \cap C_{5}$-note that (JW) is used here-and the latter because $a \in C_{3}$. So if $x_{i} \in D$ then $w=1$.

This means that we can take $x_{1}=a, x_{2}=b$. But if $x_{3}=a$ then (AB) gives $w=1$; so $x_{3}=b$. Then

$$
\begin{array}{rlrl}
w & =[a, b, b, b, a, a] & \\
& =[a, b, b ; a, b ; a]^{-1} & & \left(\mathrm{JW} ; a \in C_{3}\right) \\
& =[a, b, a ; a, b, b][a, b, b, a ; a, b]^{-1} & & (\mathrm{JW}) \\
& =[a, b, a ;[a, b], b] & & \left(a \in C_{3}\right) \\
& =[a, b, a ; a, b ; b][a, b, a, b ; a, b]^{-1} & & (\mathrm{JW}) \\
& =1 & & \left(\mathrm{AB} ; a \in C_{3}\right) .
\end{array}
$$


(ii) Suppose that $G=C_{2} \cup C_{4} \cup C_{5}$. Put $D=C_{2} \cap C_{4} \cap C_{5}, C_{2}=\langle a, D\rangle, C_{4}=\langle b, D\rangle$, $C_{5}=\langle a b, D\rangle$. We take $x_{3}=b, x_{5}=a, x_{6}=a$.

Note that since

$$
\left[x_{1}, x_{2}, b\right] \equiv\left[b, x_{2}, x_{1}\right]\left[b, x_{1}, x_{2}\right]^{-1} \bmod \gamma_{4}
$$

we have $w=1$ if $x_{1} \in D$ and $x_{2} \in D$; or if $x_{1} \in D$ and $x_{2}=a$. Indeed we can suppose that $x_{1} \in D$ or $x_{1}=a$, and that $x_{2}=b$.

Let us next consider $x_{4}$. If $x_{4} \in D$ then

$$
\begin{aligned}
w & =\left[x_{1}, b, b, x_{4}, a, a\right] & & \\
& =\left[x_{1}, b, b ; x_{4}, a ; a\right]\left[x_{1}, b, b, a, x_{4}, a\right] & & (\mathrm{JW}) \\
& =\left[x_{1}, b, b ; x_{4}, a ; a\right] & & \left(x_{4} \in C_{4}\right) \\
& =\left[x_{4}, a, a ; x_{1}, b, b\right]^{-1}\left[x_{1}, b, b, a ; x_{4}, a\right] & & (\mathrm{JW}) \\
& =1 & & \left(a \in C_{2} ; x_{4} \in C_{4} \cap C_{5}\right) .
\end{aligned}
$$

Next suppose that $x_{4}=a$. If $c$ and $d$ are commutators of weight 2 then modulo $\gamma_{6}$ we have

$$
[c, d, a] \equiv[d, a, c]^{-1}[c, a, d]
$$

and in our case, with $a \in C_{2}$, we have $w=1$. We conclude therefore that we can take $x_{4}=b$ without losing any generality.

Finally we can take $x_{1}=a$ by the following reasoning. If (LN) is applied to $\left[x_{1}, b, b, b\right.$, a] then this element becomes a product (modulo $\gamma_{6}$ ) of commutators of the form $[a, \ldots]^{ \pm 1}$, all of which are trivial when both $b$ and $x_{1}$ lie in $C_{4}$. Therefore $x_{1} \notin D$, and as above we have $x_{1}=a$. Then

$$
\begin{aligned}
w & =[a, b, b, b, a, a] & & \\
& =[a, b, b ; a, b ; a]^{-1}[a, b, b, a, b, a] & & (\mathrm{JW}) \\
& =[a, b, a ; a, b, b][a, b, b, a ; a, b]^{-1} & & (\mathrm{JW}) \\
& =1 & & \left(a \in C_{2}\right) .
\end{aligned}
$$

Note that $[a, b, b, a] \equiv 1$ because $a \in C_{2}$.

(iii) The cases in which $C_{4}$ is redundant and $C_{5}$ is redundant may be combined. Make the obvious definitions of $D$ and put $C_{2}=\langle a, D\rangle, C_{3}=\langle b, D\rangle$. We take $x_{3}=b, x_{4}=a$; and applying (JW) twice to

$$
\left[x_{1}, x_{2} ; a, b\right]\left[a, b ; x_{1}, x_{2}\right] \equiv 1 \bmod \gamma_{5}
$$

we obtain

$$
\left[x_{1}, x_{2}, a, b\right]\left[x_{1}, x_{2}, b, a\right]^{-1}\left[a, b, x_{1}, x_{2}\right]\left[a, b, x_{2}, x_{1}\right]^{-1} \equiv 1 .
$$

It is clear that if $x_{1} \in D$ then $w=1$. So by (LN), every entry of $w$ may be chosen from $\{a, b\}$. But in that case $[a, b, b, a] \equiv[a, b, a, b] \equiv 1$, and we are finished.

4. Proof of the theorem: the irredundant case. In this section we suppose that $G$ is 
a finite $p$-group with $b(G)=4, c(G)=6$, and $G=C_{2} \cup C_{3} \cup C_{4} \cup C_{5}$ as above, but $G$ is not the union of any three of the $C_{i}$. Put $D=C_{2} \cap C_{3} \cap C_{4} \cap C_{5}$. We have to consider the possibilities for $G / D$ and, just as important, for each $C_{i} / D$.

Fortunately the structure of a group covered by four proper subgroups has been given by Greco [3] and by Neumann [8]. For convenience we use the latter reference. Thus either $p=2$ and $|G: D|=8$, or $p=3$ and $|G: D|=9$.

Because the results of [8] do not give us the coverings of $G / D$ in the various cases we shall need a little elaboration. Let $H$ be a finite $p$-group which is irredundantly the union of its proper subgroups $S_{1}, S_{2}, S_{3}, S_{4}$ whose intersection is trivial. Suppose first that $p=2$ and that $H$ is elementary abelian, $H=\langle a, b, c\rangle$. We may choose $a, b, c$ so that $S_{1}=\langle a, b\rangle$ and $S_{2}=\langle a, c\rangle$ (see the table on p. 239 of [8]). Some juggling shows that in case (i) of that table we can choose $a, b, c$ so that

$$
S_{1}=\langle a, b\rangle, \quad S_{2}=\langle a, c\rangle, \quad S_{3}=\langle b, c\rangle, \quad S_{4}=\langle a b c\rangle .
$$

Case (ii) which is simpler yields

$$
S_{1}=\langle a, b\rangle, \quad S_{2}=\langle a, c\rangle, \quad S_{3}=\langle b c\rangle, \quad S_{4}=\langle a b c\rangle .
$$

Another possibility is that $H$ is abelian of order 8 and $H=\langle a, b\rangle$ with $a^{4}=b^{2}=1$. Though case (i) does not occur now, case (ii) gives

$$
S_{1}=\langle a\rangle, \quad S_{2}=\langle a b\rangle, \quad S_{3}=\langle b\rangle, \quad S_{4}=\left\langle a^{2} b\right\rangle .
$$

Finally we may have $p=3$ and $H=\langle a, b\rangle$ of order 9 with

$$
S_{1}=\langle a\rangle, \quad S_{2}=\langle a b\rangle, \quad S_{3}=\left\langle a^{2} b\right\rangle, \quad S_{4}=\langle b\rangle .
$$

Our next move is to cut down the number of possibilities embodied in (1)-(4) by borrowing some arguments of Gallian [2]. Suppose that $x$ is an element of $G$ such that $x \notin C_{4} \cup C_{5}$. Since $x \notin C_{4}, b\left(x \gamma_{5}\right)<b\left(x \gamma_{6}\right)$; since $x \notin C_{5}, b\left(x \gamma_{6}\right)<b(x) \leq 4$-here we are using that part of Lemma 2.1 of [6] stated as Lemma 2 of [2]. So $b\left(x \gamma_{5}\right) \leq 2$. It follows that $c\left(\left(x \gamma_{5}: x \notin C_{4} \cup C_{5}\right\rangle\right) \leq 3$, by Theorem 2 of [5] also to be found as Lemma 1 of [2]. Because $K=\left\langle x \in G: x \notin C_{4} \cup C_{5}\right\rangle$ therefore has $c(K) \leq 5$, we see that $K \neq G$. (In rough terms we can say that " $C_{4}$ and $C_{5}$ must not be too small.")

This at once implies that (4) does not occur. Neither does (3), though this is less obvious. First we note that if $4 \leq i \leq 5$ then $C_{i} / D$ must have order 4 . Next we lose no generality in taking $b \in C_{2}, a^{2} b \in C_{3}, a \in C_{4}, a b \in C_{5}$. As usual in these calculations we put

$$
w=\left[x_{1}, x_{2}, x_{3}, x_{4}, x_{5}, x_{6}\right] \neq 1 \text {, }
$$

and we assume that $x_{3}=a, x_{5}=b$. Suppose every $x_{i}$ lies in $\{a, b\}$ and take $x_{1}=a, x_{2}=b$. Since $a b \in C_{5}$, we lose no generality in putting $x_{6}=b$. Thus

$$
w=\left[a, b, a, x_{4}, b, b\right]
$$

If $x_{4}=b$ we find that $w=1$ by applying (AB) to $\left[a, b, a, x_{4}\right]$ and noting that $b \in C_{2}$. If 
$x_{4}=a$ then

$$
\begin{aligned}
w & =[a, b, a ; a, b ; b][a, b, a, b, a, b] & & (\mathrm{JW}) \\
& =[a, b, a ; a, b ; b] & & \left(\mathrm{AB} ; b \in C_{2}\right) \\
& =[a, b, b ; a, b, a]^{-1}[a, b, a, b ; a, b] & & (\mathrm{JW}) \\
& =1 & & \left(\mathrm{AB} ; b \in C_{2}\right) .
\end{aligned}
$$

Thus the final stage of the argument requires $x_{1} \in D$. If $x_{4}=b$ then

$$
\left[x_{1}, x_{2}, a, b\right]\left[x_{1}, x_{2}, b, a\right]^{-1}\left[a, b, x_{1}, x_{2}\right]\left[a, b, x_{2}, x_{1}\right]^{-1} \equiv 1 \bmod \gamma_{5}
$$

gives $w=1$, while if $x_{4}=a$ then

$$
\begin{aligned}
w & =\left[x_{1}, x_{2}, a ; a, b ; a\right] & & \left(\mathrm{JW} ; a \in C_{4}\right) \\
& =\left[a, b ; x_{1}, x_{2} ; a ; a\right]^{-1}\left[a, b, a ; x_{1}, x_{2} ; a\right] & & (\mathrm{JW}) \\
& =1 & & \left(x_{1} \in D\right) .
\end{aligned}
$$

So (3) does not occur.

In cases (1) and (2) $G$ has at least three generators, and we make a remark which will be important later: we can assume that the case when $G$ is generated by two elements has been disposed of.

Gallian's argument shows that in both (1) and (2) we can take $C_{4}=\langle a, b, D\rangle$, $C_{5}=\langle a, c, D\rangle$. Correspondingly we can assume that $x_{5}=c, x_{6}=b$ in the usual way. In fact, we get more by applying (LN) to $\left[x_{1}, \ldots, x_{4}, c\right]$; we may suppose that in addition $x_{1}=c$. So

$$
w=\left[c, x_{2}, x_{3}, x_{4}, c, b\right] \text {. }
$$

We proceed to dispose of the subcase of (1) in which $C_{2}=\langle b, c, D\rangle, C_{3}=\langle a b c, D\rangle$. We can suppose that $x_{3}=a$. Since

$$
[c, b, a][b, a, c][a, c, b] \equiv 1 \bmod \gamma_{4},
$$

the composition of $C_{2}$ shows that $[c, b, a] \equiv 1 \bmod \gamma_{4}$, and so if $x_{2}=b$ then $w=1$. So

If $x_{4}=b$ then

$$
w=\left[c, a, a, x_{4}, c, b\right] \text {. }
$$

$$
\begin{aligned}
{[c, a, a, b, c] } & \equiv[c, a ; a ; b, c] \\
& \equiv[b, c, a ; c, a][b, c ; c, a ; a]^{-1} \\
& \equiv 1
\end{aligned}
$$

because $[b, c, a] \equiv 1$ and because $a \in C_{4}$. If $x_{4}=c$ then $w=1$ by (AB) and $c \in C_{2}$. Therefore the fact that $a b c \in C_{3}$ shows that if $x_{4}=a$ then $w=1$.

The subcase does not occur then, and we recapitulate by rewriting (1) and (2) as

$$
\begin{aligned}
& C_{2}=\langle a b c, D\rangle, \quad C_{3}=\langle b, c, D\rangle, \quad C_{4}=\langle a, b, D\rangle, \quad C_{5}=\langle a, c, D\rangle, \\
& C_{2}=\langle a b c, D\rangle, \quad C_{3}=\langle b c, D\rangle, \quad C_{4}=\langle a, b, D\rangle, \quad C_{5}=\langle a, c, D\rangle .
\end{aligned}
$$


Note that interchanging $C_{2}$ and $C_{3}$ in $\left(2^{\prime}\right)$ gives nothing essentially new.

In $\left(1^{\prime}\right)$ we have $w=\left[c, x_{2}, x_{3}, a, c, b\right]$. First suppose that $x_{2}=a$. If $x_{3}=c$ then (AB) gives $w=1$. If $x_{3}=b$ then

$$
\begin{aligned}
w & =[c, a, b, a, c, b] & & \\
& =[c, a ; b, a ; c ; b] & & \left(\mathrm{JW} ; b \in C_{3}\right) \\
& =[b, a, c ; c, a ; b]^{-1}[c, a, c ; b, a ; b] & & (\mathrm{JW}) \\
& =[b, a, c, a, c, b] & & \left(\mathrm{JW} ; a \in C_{4} ; b \in C_{4}\right) .
\end{aligned}
$$

However both $[b, a, a, a, c, b]$ and $[b, a, b, a, c, b]$ are trivial, because (LN) can be applied to $[b, a, x, a, c]$ and the composition of $C_{4}$ used. The fact that $a b c \in C_{2}$ then shows that $w=1$. Next we suppose that $x_{3}=a$. In that case $a b c \in C_{2}$ implies that $w=1$. So if $x_{2}=a$ then $w=1$.

If $x_{2}=b$ then we apply (LN) to $\left[c, b, x_{3}, a\right]$; when $x_{3}=b$ or $c$ we have $\left[c, b, x_{3}, a\right] \equiv 1$ because of $C_{3}$, and so $w=1$. It follows that if $x_{3}=a$ then $w=1$ because $a b c \in C_{2}$.

To complete the case $\left(1^{\prime}\right)$ we discuss the implications of taking $x_{2} \in D$. Mod $\gamma_{5}$,

$$
\begin{aligned}
{\left[c, x_{2}, x_{3}, a\right] } & \equiv\left[c, x_{2}, a, x_{3}\right]\left[c, x_{2} ; x_{3}, a\right] \\
& \equiv\left[c, x_{2}, a, x_{3}\right]
\end{aligned}
$$

because $x_{2} \in \cap C_{3}$ gives $\left[x_{3}, a ; c, x_{2}\right] \equiv 1$; so if $x_{3}=b$ or $c$ then $w=1$ in view of $C_{3}$, and consequently if $x_{3}=a$ then $w=1$ because $a b c \in C_{2}$. Thus if $x_{2} \in D$ then $w=1$.

In case $\left(2^{\prime}\right)$ we have $w=\left[c, x_{2}, x_{3}, x_{4}, c, b\right]$. First suppose that $x_{2}=a$. It suffices to prove that $w=1$ whenever $x_{3}, x_{4} \in\{a, c\}$ in view of $C_{2}$ and $C_{3}$. But this is obvious.

Secondly suppose that $x_{2}=b$. If $x_{4}=b$ then $c(G)<6$ by a remark above (note that we may assume $x_{3}=b$ or $c$ ), so we take $x_{4}=a$. Mod $\gamma_{6}$ we have

$$
\begin{array}{rlrl}
{\left[c, b, x_{3}, a, c\right]} & \equiv\left[c, b, x_{3} ; a, c\right] & \left(\mathrm{JW} ; a \in C_{4}\right) \\
& \equiv\left[a, c, x_{3} ; c, b\right]\left[a, c ; c, b ; x_{3}\right]^{-1} & & (\mathrm{JW}) \\
& \equiv\left[a, c, x_{3} ; c, b\right] & & \left(x_{3} \in C_{4}\right),
\end{array}
$$

provided we assume (as we may) that $x_{3}=a$ or $b$. So

$$
w=\left[a, c, x_{3}, b, c, b\right]^{-1}
$$

since $b \in C_{4}$, and $w=1$ follows because the case $x_{2}=a$ was dealt with in the previous paragraph.

Thirdly suppose that $x_{2} \in D$. We may take

$$
w=\left[c, x_{2}, x_{3} ; x_{4}, c ; b\right]
$$

because $x_{4}=a$ or $b$ in view of $C_{3}$, and so $x_{4} \in C_{4}$. Next we apply (LN) to $\left(c, x_{2}, x_{3},\left[x_{4}, c\right]\right]$. The fact that $x_{2} \in D$ then shows that $w=1$.

This completes the case $\left(2^{\prime}\right)$ and with it the proof of the theorem. 


\section{REFERENCES}

1. W. Burnside, Theory of Groups of Finite Order, (Dover, 1955).

2. Joseph A. Gallian, On the breadth of a finite p-group, Math. Z. 126 (1972), 224-226.

3. Donato Greco, I gruppi finiti che sono somma di quattro sottogruppi, Rend. Accad. Sci. Fis. Mat. Napoli (4) 18 (1951), 74-85.

4. Seymour Haber and Azriel Rosenfeld, Groups as unions of proper subgroups, Amer. Math. Monthly 66 (1959), 491-494.

5. Hans-Georg Knoche, Über den Frobenius' schen Klassenbegriff in nilpotenten Gruppen II. Math. Z. 59 (1953), 8-16.

6. C. R. Leedham-Green, Peter M. Neumann and James Wiegold, The breadth and the class of a finite p-group, J. London Math. Soc. (2) 1 (1969), 409-420.

7. I. D. Macdonald, The breadth of finite p-groups I. Proc. Royal Soc. Edinburgh Sect A 78 (1977), 31-39.

8. B. H. Neumann, Groups covered by finitely many cosets, Publ. Math. Debrecen 3 (1954), 227-242.

Department of Mathematics

UNIVERSITY OF STIRLING

STIRLING

SCOTLAND 